\title{
A Spatial Registration Toolbox for Structural MR Imaging of the Aging Brain
}

\author{
Marco Ganzetti ${ }^{1} \cdot$ Quanying Liu ${ }^{1,2} \cdot$ Dante Mantini ${ }^{1,2,3} \cdot$ Alzheimer's Disease Neuroimaging Initiative
}

(C) Springer Science+Business Media, LLC, part of Springer Nature 2018

\begin{abstract}
During aging the brain undergoes a series of structural changes, in size, shape as well as tissue composition. In particular, cortical atrophy and ventricular enlargement are often present in the brain of elderly individuals. This poses serious challenges in the spatial registration of structural MR images. In this study, we addressed this open issue by proposing an enhanced framework for MR registration and segmentation. Our solution was compared with other approaches based on the tools available in SPM12, a widely used software package. Performance of the different methods was assessed on 229 T1-weighted images collected in healthy individuals, with age ranging between 55 and 90 years old. Our method showed a consistent improvement as compared to other solutions, especially for subjects with enlarged lateral ventricles. It also provided a superior inter-subject alignment in cortical regions, with the most marked improvement in the frontal lobe. We conclude that our method is a valid alternative to standard approaches based on SPM12, and is particularly suitable for the processing of structural MR images of brains with cortical atrophy and ventricular enlargement. The method is integrated in our software toolbox MRTool, which is freely available to the scientific community.
\end{abstract}

Keywords Magnetic resonance imaging $\cdot$ Spatial registration $\cdot$ Normalization $\cdot$ Segmentation $\cdot$ Aging $\cdot$ MRTool $\cdot$ Large ventricles

Alzheimer's Disease Neuroimaging Initiative (ADNI) is a Group/ Institutional Author

Data used in preparation of this article were obtained from the Alzheimer's Disease Neuroimaging Initiative (ADNI) database (adni. loni.usc.edu). As such, the investigators within the ADNI contributed to the design and implementation of ADNI and/or provided data but did not participate in analysis or writing of this report. A complete listing of ADNI investigators can be found at: http://adni.loni.usc.edu/wpcontent/ uploads/how_to_apply/ADNI_Acknowledgement_List.pdf

Electronic supplementary material The online version of this article (https://doi.org/10.1007/s12021-018-9355-3) contains supplementary material, which is available to authorized users.

Marco Ganzetti

marco.ganzetti@kuleuven.be

1 Laboratory of Movement Control and Neuroplasticity, KU Leuven, Leuven, Belgium

2 Neural Control of Movement Lab, ETH Zurich, Zurich, Switzerland

3 Department of Experimental Psychology, Oxford University, Oxford, UK

\section{Introduction}

The occurrence of widespread anatomical changes in the normal aging brain is a well-established mechanism, even in the absence of dementia or other neurological disorders. Converging evidence for age-related alterations suggested atrophy to involve the whole brain, but to be relatively more pronounced in the neocortex and the hippocampus (Mcdonald et al. 2009). Furthermore, an expansion of lateral ventricles is typically found in older individuals (Whitwell et al. 2008; Apostolova et al. 2012). Because these age-related changes vary across the brain, being able to accurately quantify regionally-specific effects over the lifespan has been a topic of considerable interest, for reasons that are both fundamental (e.g., exploring structure-function interactions by linking volumetric changes to behavioral scores) and clinical (e.g., detecting regional alterations to aid in diagnosis).

Common reference frames have been established to consistently and precisely evaluate inter-subject anatomical correspondences across structural images collected with magnetic resonance (MR). This is done by transforming the brain to a common neuroanatomical space (e.g. the stereotactic space of the Montreal Neurological Institute, MNI), 
which is defined by means of a template image (Brett et al. 2002; Crivello et al. 2002; Martino et al. 2013). Such process is commonly referred to as spatial registration or normalization. Many studies investigate anatomical differences between groups by transforming MR images to template space and focusing on brain structures of interest (e.g. gray matter, GM and white matter, WM), which need to be segmented. Therefore, most advanced algorithms adopt a combined approach to spatial registration and segmentation. Given the fact that an accurate tissue classification is often associated with precise registration (Van Leemput et al. 1999a), segmentation is frequently implemented within a unified framework (Ashburner and Friston 2005; Pohl et al. 2005). The use of a priori information in the form of tissue probability maps (TPMs) is a standard procedure within unified registration-segmentation frameworks. It is worth noting that TPMs are representative of a specific group of individuals from which they are generated, and need to be defined in the same neuroanatomical space as the template.

Nowadays there are multiple alternatives for spatial registration and segmentation of MR images (Crivello et al. 2002; Klein et al. 2009) and, arguably, one of the most robust and widely used software package is SPM12. Its nonlinear registration and segmentation methods, jointly implemented in a unified framework (Ashburner and Friston 2005; Pohl et al. 2005), have been successfully used in numerous investigations (Dewey et al. 2010; Colloby et al. 2011; Izquierdo-Garcia et al. 2014). Several comparative studies have been conducted to examine the accuracy of SPM12 on MR images collected in young individuals (Wilke et al. 2002; Klein et al. 2009; Han et al. 2012; Martino et al. 2013; Pu et al. 2013), but not yet in elderly populations. There is however a general consensus on the fact that age-related and pathological changes have a negative impact on the performance of the method adopted (Dade et al. 2004; Hoeksma et al. 2005; Allen et al. 2008; Kennedy et al. 2009; Peelle et al. 2012; Eloyan et al. 2014). This is still the case when population-specific templates are used (Peelle et al. 2012). Notably, the large inter-individual variability that characterizes MR images from older individuals poses serious challenges in the definition of adequate TPMs. This may impair the registration of anatomical structures with ambiguous intensity patterns (Pohl et al. 2005). On the other hand, a possible misalignment between anatomical structures of individual and template brains may prevent the detection of distinct tissue boundaries, thereby complicating segmentation (Fischl et al. 2002). A possible way for alleviating these problems that may affect structural MR registration is the use brain extraction (Fein et al. 2006; Acosta-Cabronero et al. 2008; Fischmeister et al. 2013). This permits registration algorithms to explicitly define the boundaries between the brain and other head tissues. However, even when using brain extraction, the definition of gray and white matter remains complicated, particularly in the case of MR images from older individuals.

In this study, we address the problem described above by introducing an original method to reduce confounding effects of inter-individual structural variability in elderly populations. The method is implemented as an enhanced processing framework, which makes use of the image processing tools available in SPM12. Its performance is assessed on a large number of MR images collected in elderly subjects, and typically characterized by ventricular enlargement and cortical atrophy. Other approaches based on SPM12 are also evaluated, to provide a benchmark measure of registration and segmentation accuracy. The use of SPM12 is based on considerations of convenience and does not imply any statement of its advantages over other popular registration and segmentation methods (see Klein et al. 2009 for a comprehensive review).

\section{Methods}

In this section, we provide a comprehensive overview of our approach for spatial registration and segmentation of structural MR images (Fig. 1). For the sake of simplicity and consistency, we will refer to it with the term 'MRTool' which is also the name of our software toolbox that implements the proposed spatial registration method (www.nitrc.org/projects/mrtool). Then we outline the validation scheme in terms of brain imaging data, performance measures and methods used as benchmark.

\section{Method Description}

The approach here presented is an enhanced version of the one originally proposed by Ashburner and Friston (2005) and currently implemented in SPM12 (http://www.fil.ion. ucl.ac.uk/spm/software/spm12/).The processing workflow is composed of several steps (Fig. 1), and requires a raw (unprocessed) T1-weighted (T1-w) image as input. The output is a normalized version of the same image in template space with the respective segmented tissue classes both in subject and template space. The segmented tissues are: white matter (WM), gray matter (GM), and cerebrospinal fluid (CSF). This ensures compatibility with other brain imaging analysis modules based on SPM12. 


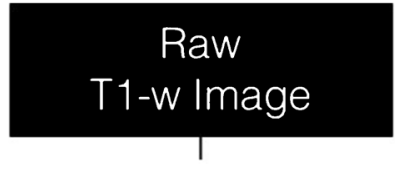

rigid-body registration

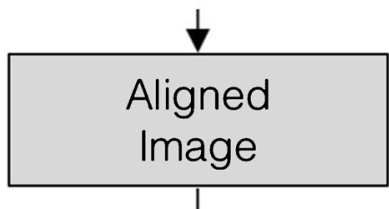

INU correction

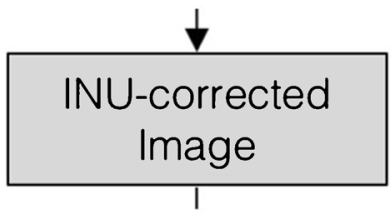

brain extraction
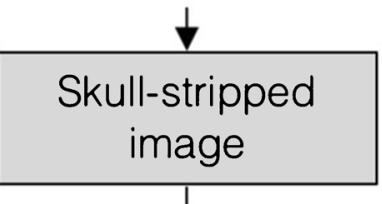

intensity distribution extraction

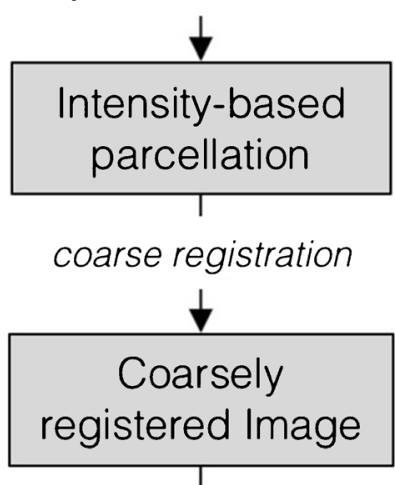

unified registration-segmentation

\section{Normalized} T1-w Image

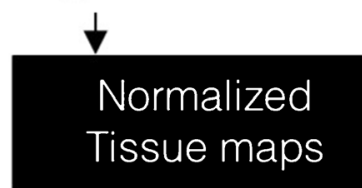

Fig. 1 MRTool registration-segmentation framework. The diagram provides an overview of the sequential framework for the spatial registration and segmentation of structural T1-weighted images. An initial intensity inhomogeneity (INU) correction is performed on the raw image. Afterwards, a brain extraction procedure is applied to the volume and the resulting image nonlinearly registered using the 'Old Normalization' module of SPM12 roughly matching the subject brain to the template's. Finally, the unified registration-segmentation approach of SPM12 is run on the 'coarsely registered image' to produce the normalized image and respective tissue probability maps (TPM) of gray matter (GM), white matter (WM), and cerebrospinal fluid (CSF)

\section{Description of the Main Processing Steps}

First of all, the intensity non-uniformity (INU) artefact is removed from the structural MR image according to the method implemented in SPM12. The selection of optimal input parameters for INU correction can be performed using a dedicated tool that we developed in our previous work (Ganzetti et al. 2015, 2016). The second analysis step comprises a rigid-body alignment of the INU-corrected image to the template (Collignon et al. 1995). This preparatory step allows the brain to acquire similar orientation to the template, thus facilitating subsequent registration steps. At this point, the aligned image undergoes a brain extraction (see Supplementary Fig. 1) and the resulting skull-stripped image is given as input to next registration procedure. Using the 'Segmentation' module in SPM12, the skull-stripped image is segmented into GM, WM and CSF. The intensity distribution of the three segmented tissues is extracted from the skullstripped image using the respective masks. Afterwards, cut-off values separating the distributions of values for GM, WM and CSF are automatically extracted and used for intensity-based image parcellation. This produces a clustered image (Fig. 2). The same procedure is repeated for the T1-w template image in SPM12 to generate a clustered template. The clustered image is then nonlinearly registered to the clustered template using the 'Old normalization' module of SPM12 (Ashburner and Friston 1999), and the warping field in output from this transformation is applied to skull-stripped image. The primary aim of this registration is to (at least partially) compensate for potentially enlarged ventricles. A final registration step was run using the 'Normalization' module of SPM12. At this point, the two transformations were combined using the 'Deformation' toolbox in SPM12. Spatial deformations can be thought as composite transformation with the same domain of the first and codomain of the last. Consequently, the composite field was applied to the INUcorrected skull-stripped image, to produce its normalized version in template space. Eventually, tissue probability maps in template space are produced by segmenting the normalized image using the 'Segmentation' module. These are then mapped back to original space by applying the inverse deformation, as calculated using the 'Deformation' toolbox.

\section{Method Validation}

\section{Subjects}

A total of 229 healthy subjects (112 females) was extracted from the publically available Alzheimer's Disease Neuroimaging Initiative (ADNI) dataset (http://adni.loni.usc.edu). ADNI is a longitudinal, multicenter study with the goal of developing clinical, imaging, genetic, and biochemical biomarkers for the 


\section{Intensity-based parcellation}

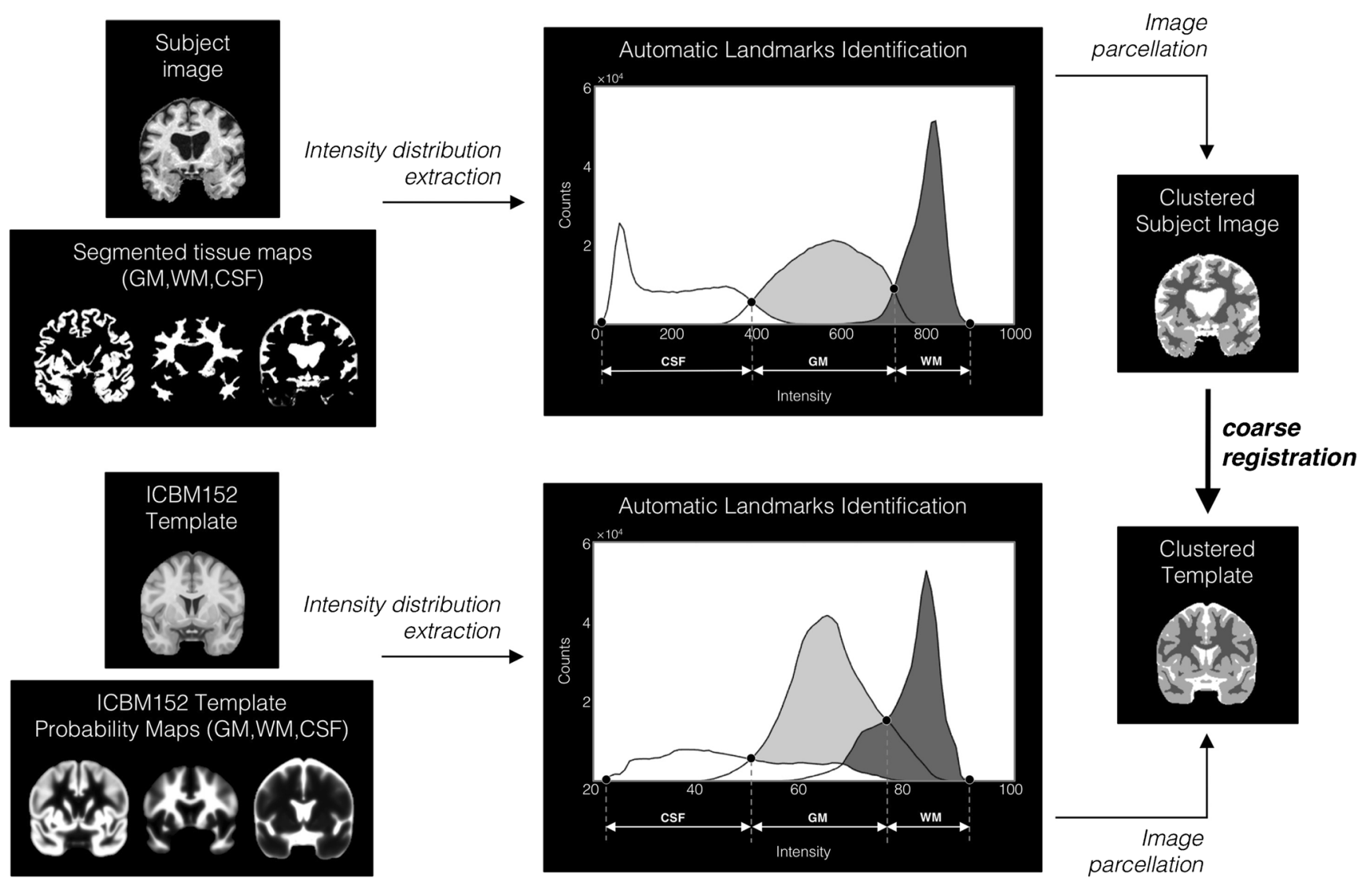

Fig. 2 Intensity-based parcellation. The diagram provides an overview of the intensity-based parcellation scheme for the generation of the nonlinear transformation associated with the 'coarse registration'. Both the subject skull-stripped image and the T1-weigthed template in MNI space, with the respective tissue segmentation (GM, WM, CSF) and probability maps (TPM) are given as input. Afterwards, using the segmented tissue maps, the three intensity distributions are extracted from the structural image and the automatic landmark identification procedure is performed to generate a clustered image. The same parcellation procedure is applied to the template and a nonlinear deformation is computed to match these two clustered images

information regarding acquisition parameters at each different site, please refer to (Petersen et al. 2010; Weiner et al. 2012).

\section{Evaluation of Brain Imaging Data}

To assess the performance of spatial registration we computed two quantitative indices. First, the Pearson correlation coefficient $(\mathrm{CC})$ was used to measure the similarity between the normalized T1-w image (I) and the template (T):

$$
C C(I, T)=\frac{\sum_{k \rightarrow 1}^{n}\left(I_{k}-\bar{I}\right) \cdot\left(T_{k}-\bar{T}\right)}{\sqrt{\sum_{k \rightarrow 1}^{n}\left(I_{k}-\bar{I}\right)^{2} \cdot \sum_{k \rightarrow 1}^{n}\left(T_{k}-\bar{T}\right)^{2}}}
$$

where $\mathrm{n}$ is the total number of voxels $\mathrm{k}$ in the skull stripped images. CC is a similarity measure commonly used when comparing two images from the same modality (Lemieux et al. 1994; Dong and Boyer 1995). 
The mutual information (MI) between the two images was also calculated, to take into account also the nonlinear part of the relationship (Wells 3rd et al. 1996):

$$
M I(I, T)=\sum_{i \in I} \sum_{t \in T} p(i, t) \cdot \log \left(\frac{p(i, t)}{p(i) \cdot p(t)}\right)
$$

where $\mathrm{p}(\mathrm{i}, \mathrm{t})$ is the joint probability distribution function of the image I and the template $T$, while $p(i)$ and $p(t)$ are the marginal probability distribution functions of I and T, respectively (Maes et al. 1997). For both metrics, statistical significance between methods was tested using the non-parametric Wilcoxon signedrank test $(*: \mathrm{p}<0.05, * *: \mathrm{p}<0.01, * * *: \mathrm{p}<0.001)$. Effect size was calculated for each paired t-test by dividing the test statistic by the square root of the number of subjects. An effect size of 0.1 is considered a small effect, 0.3 moderate, and 0.5 is considered a large effect size (Pallant 2013).

In parallel to the spatial registration, we also assessed the quality of the segmentation. Indeed, in SPM12 as well as with our approach, spatial registration and segmentation are jointly executed within the same processing framework. Therefore, evaluating the segmentation can be considered as a complementary manner of assessing the registration accuracy. We adopted the Dice Similarity index (DSI), which is a wellknown metric for evaluating segmentation outputs (Van Leemput et al. 1999b; Shattuck et al. 2001; Fischmeister et al. 2013), to measure the quality of the segmentation. DSI was calculated as follows:

$\operatorname{DSI}(S 1, S 2)=2 \cdot \frac{\sum_{k \rightarrow 1}^{n} S 1_{k} \cdot S 2_{k}}{\sum_{k \rightarrow 1}^{n} S 1_{k}+\sum_{k \rightarrow 1}^{n} S 2_{k}}$

where $\mathrm{S}_{\mathrm{k}}$ is the individual TPM of either GM or WM, normalized to template space and thresholded at 0.5. DSI values range from zero to one, where a higher DSI indicates complete overlap. A complementary analysis was carried out at a voxelwise level for the normalized segmented maps of GM and WM. Specifically, a one-sample nonparametric t-test was performed using FSL (https://fsl.fmrib.ox.ac.uk/fsl/fslwiki) to assess the inter-subject variability. A direct comparison between methods was performed also in FSL by using a nonparametric paired t-test. For both analyses, the number of permutations was set at 5000. Significance threshold was set to $p$ $<0.05$, corrected for multiple comparisons using the threshold free cluster enhancement (TFCE) method.

In addition, we examined performance for different subsets of MR images, classified based on lateral ventricles volume. To this end, ventricles were manually segmented on the individual's T1-w image by an experienced radiologist using the opensource software, ITK-SNAP (http://www.itksnap.org). Ventricles volume was then calculated and expressed as percentage of the whole brain volume. In our dataset, relative ventricular volume had a wide distribution (range: $0.8-5.3 \%$, mean \pm SD: $2.56 \pm 0.92 \%$, median: $2.47 \%$ ) suggesting a possible effect of the lateral ventricles volume on registration performance (see Supplementary Fig. 2). To this end, we examined CC and MI values obtained for each method separately and fitted a linear model to the data. Also, the slopes of the regression lines generated by each fit were compared as follows:

$z=\frac{m_{1}-m_{2}}{\sqrt{S E_{m_{1}}^{2}+S E_{m_{2}}^{2}}}$

where $\mathrm{m}$ specifies the slope of the regression line and SE indicates the standard error of each slope (Kleinbaum et al. 2007).

Furthermore, we computed the DSI between the normalized tissue maps, and examined whether this measure depended on relative ventricular size in the original MR image.

\section{Comparison with Other Approaches in SPM12}

We assessed the performance of our method with respect to that of two different normalization/segmentation approaches based on SPM12. They are both commonly used by the neuroscience community. The first approach, referred to as 'SPM12', is the standard approach in SPM12. Here, INU correction, spatial registration and segmentation are jointly executed within the same unified framework (Ashburner and Friston 2005) using the 'Segmentation' module (Fig. 3). In the second approach, 'SPM12 + SS', where SS stands for skull-stripping, INU correction is first employed. Then, brain extraction is applied (for more information, see Supplementary Fig. 1). Finally, spatial registration and segmentation are performed using the 'Segmentation' module while disabling INU correction. For the two preprocessing setups described above, as well as for 'MRTool', we used the ICBM152-MNI T1-w image as template, which is the standard template image in SPM12.

\section{Results}

As a first analysis, we visually compared the registration performance of our approach, which we refer to as 'MRTool', with respect to that obtained with SPM12 using the two processing setups (see Fig. 4). We observed that spatial registration was not always accurate with 'SPM12'. A misalignment compared to the template was especially visible at the interface between brain and skull. On the other hand, a clear enhancement in spatial registration was achieved when employing 'SPM12 + SS'. As compared to this approach, 'MRTool' allowed a further improvement 
a

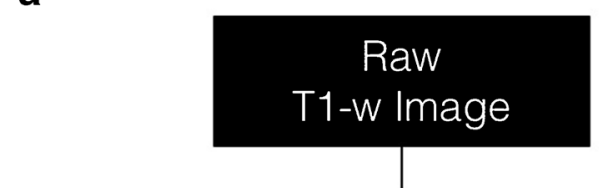

unified registration-segmentation
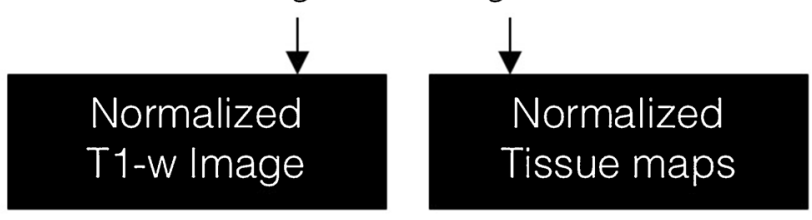

b

\section{SPM12+SS}

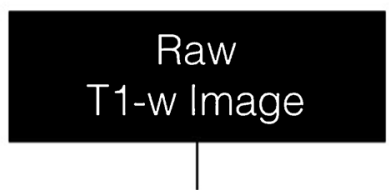

INU correction

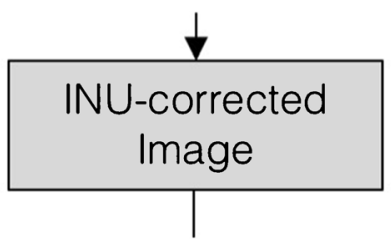

brain extraction

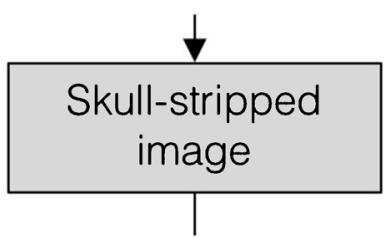

unified registration-segmentation
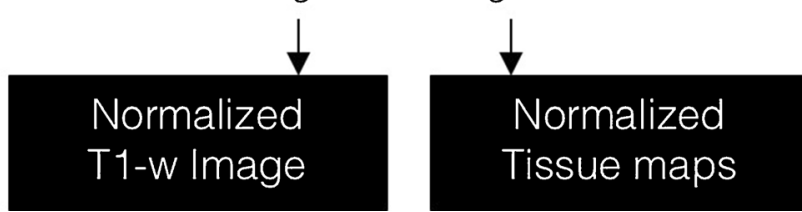

Fig. 3 Spatial registration and segmentation: processing setups in SPM12. These diagrams provide an outline of two processing setups for the normalization and segmentation of structural T1-weighted (T1w) images in SPM12. Both procedures were performed using the unified registration-segmentation framework implemented within the 'Segmentation' module of SPM12. In the first processing setup, the unified algorithm is directly applied to the raw T1-w image, including INU correction ('SPM12'). As for the second setup ('SPM12+SS'), INU correction and brain extraction are sequentially employed, and then the unified framework (without INU correction) is run

around the lateral ventricles, which more closely resembled those of the template.

To corroborate this qualitative investigation, we performed a statistical analysis using quantitative indices, such as the $\mathrm{CC}$ and MI between normalized images (Fig. 5). Specifically, CC and MI between the normalized T1-w image and the template showed a substantial improvement of 'SPM12 + SS' compared to 'SPM12'. The inter-subject variability was uneven across methods, and gradually decreasing when going from 'SPM12' to 'SPM12 + SS', and then to 'MRTool'. Although 'MRTool' was characterized by slightly larger values than 'SPM12 + SS' for both CC and MI, the difference was statistically significant $(\mathrm{p}<0.001$, effect size $>0.8$, see also Supplementary Fig. 3).

In parallel to spatial registration, the accuracy of segmentation was also evaluated. First, a visual inspection on the segmented tissue maps of GM and WM (Fig. 6) was performed on the same subjects of Fig. 4. Clear differences across subjects were present in 'SPM12'. As already observed for spatial registration (Fig. 4), the effect was particularly evident around the periventricular WM. On the other hand, a distinct improvement in the segmentation similarity was achieved with 'SPM12 + SS'. The correspondence with the TPM of the template is further increased in the case of 'MRTool', mostly in the WM.

To further extend the segmentation results to the whole dataset, we analyzed the segmented tissue maps of GM and WM using a one-sample nonparametric t-test at the single voxel level (Fig. 7 and Supplementary Fig. 4). The resulting t-score maps support our previous finding suggesting a less accurate spatial registration and segmentation of 'SPM12' as compared to 'SPM12 + SS'. Not only was this effect detected at frontal cortical (FC) regions, but also WM structures showed decreased t-scores indicating a potential misalignment across subjects. As for 'SPM12 + SS', a visible increase of t-scores was mainly found in frontotemporal WM tracts, such as forceps minor (FM), anterior thalamic radiation (ATR) and inferior longitudinal fasciculus (ILF). On the other hand, 'MRTool' unveiled a confined and homogenous pattern characterized by relatively high t-score values throughout the brain. Its advantage is mainly evident around the ventricular region, with a clear delineation of the border between the CSF and the surrounding WM (Fig. 7b, d). In particular, structures adjacent the lateral ventricles (LV) exhibited a well-delineated morphological profile with large $t$-values in correspondence of the corpus callosum (CC), both anterior (GCC) and posterior (SCC) aspect. In terms of GM (Fig. 7a, c), major improvements were within the prefrontal cortex and extend posteriorly to the dorsal portion of the frontal lobe, e.g. the precentral gyrus (PG).

Given the large inter-subject variability in brain anatomy concerning lateral ventricles size and the known relationship between ventricular enlargement and aging (Supplementary Fig. 2), we performed a more detailed analysis of the method performance with respect to ventricle size. Our analysis showed a clear, negative relationship between relative ventricle volume and spatial registration performance (Fig. 8). 'MRTool' showed higher CC and MI values with respect to 


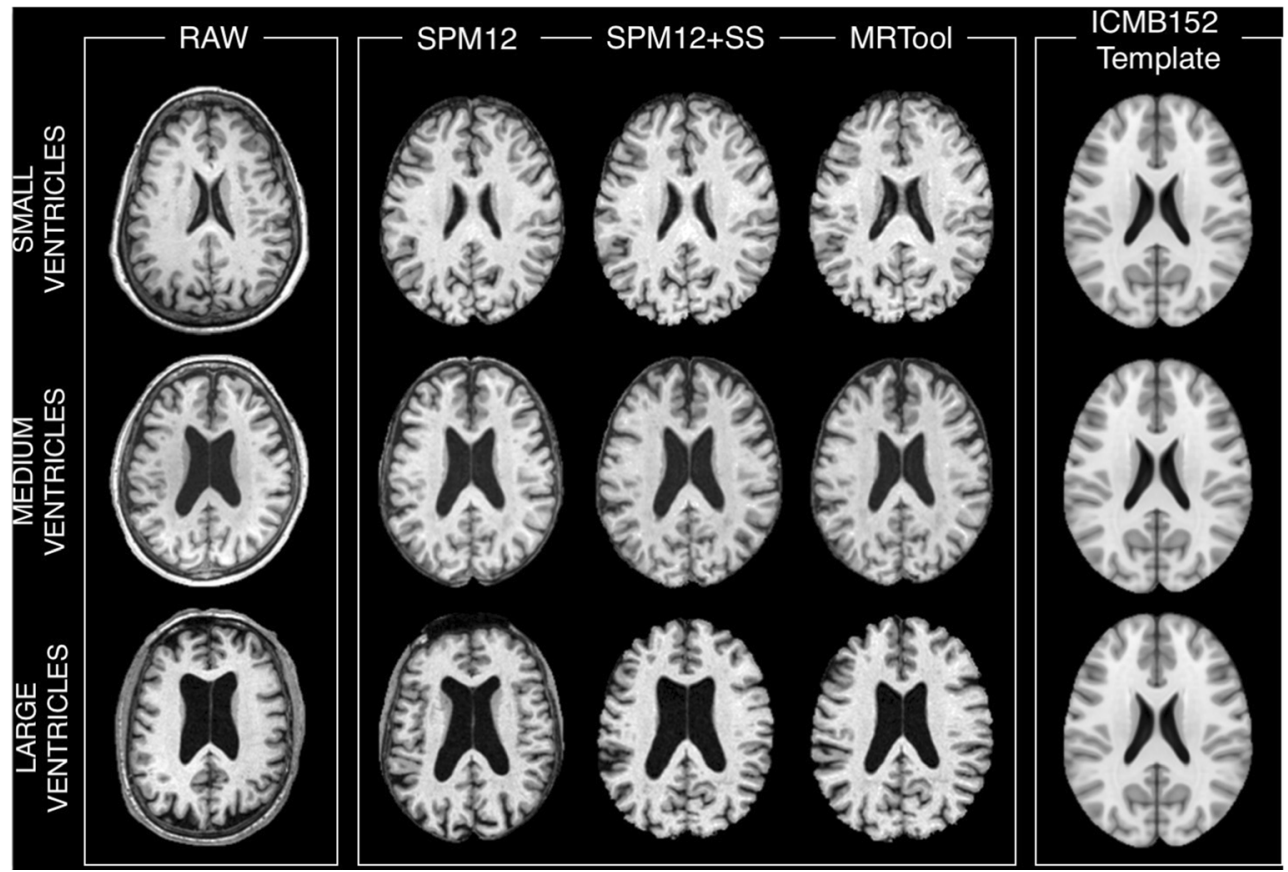

Fig. 4 Spatial registration: qualitative analysis. Normalized T1weigthed (T1-w) images (axial cut-plane) of three representative subjects (top row: female 75.4 years old, middle row: female 80.1 years old, bottom row: male 70.4 years old) with increasing lateral ventricles volume $(20 \mathrm{~mL}, 45.9 \mathrm{~mL}, 81.3 \mathrm{~mL})$ are shown. From left to right: the unprocessed raw $\mathrm{T} 1-\mathrm{w}$ image, the spatially registered image with the standard processing setup applied ('SPM12'), with the second applied ('SPM12 + SS'), the spatially registered image after implementing our method ('MRTool'), and the reference ICBM152-MNI T1-w template. To facilitate visual comparisons across methods, the same mask (derived from the MR template) was applied to all the registered images

We also conducted an analysis of inter-subject variability for the segmentation results, by computing the degree of tissue overlap between pairs of subjects (Fig. 9). 'SPM12' generally showed lower inter-subject overlap with respect to 'SPM12+ SS' and 'MRTool'. The use of 'MRTool' yielded higher DSI

b

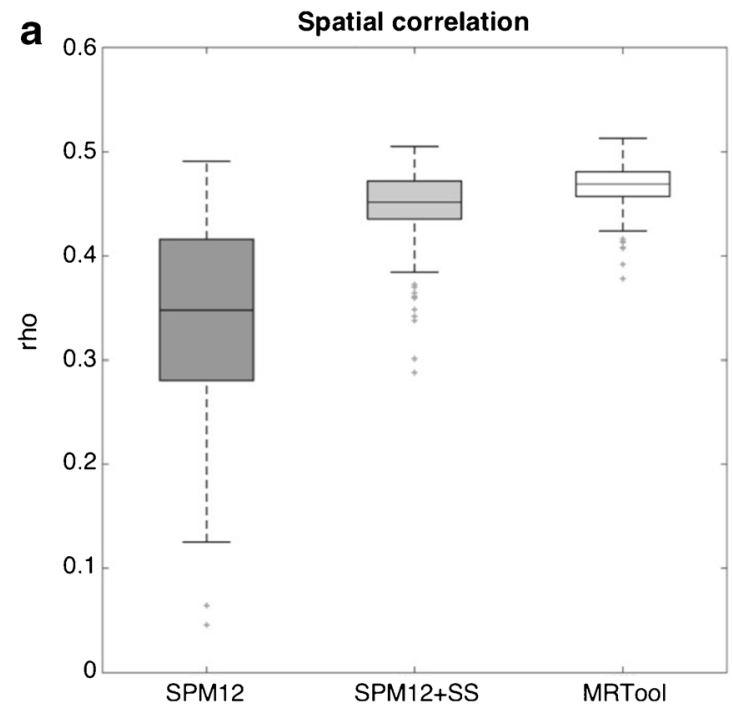

Fig. 5 Image registration assessment. These box and whisker plots show the voxel-wise CC (a) and MI (b) between the normalized T1-weighted (T1-w) image and the reference ICBM152-MNI T1-w template for the two

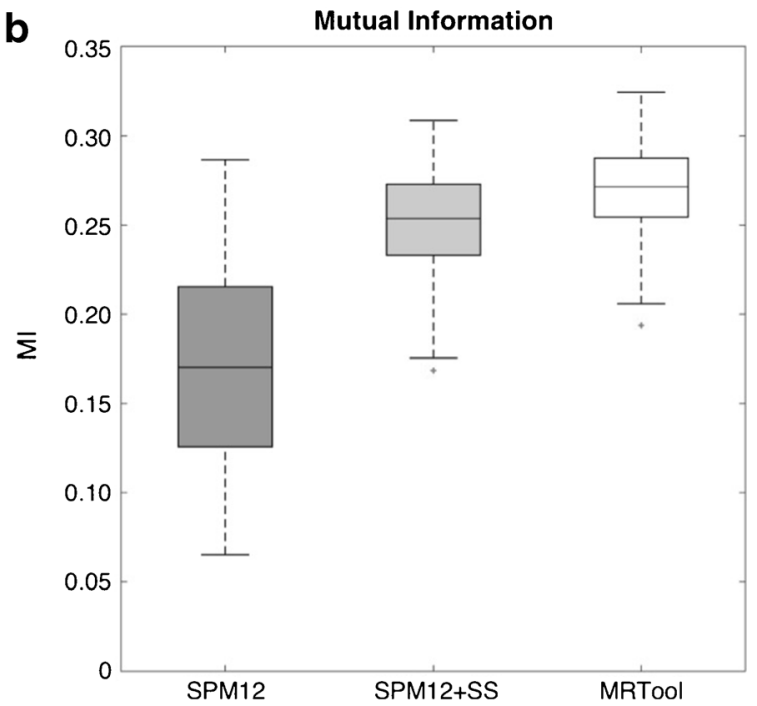

SPM12 setups ('SPM12', 'SPM12 + SS') and our approach ('MRTool'). The inter-subject variability, expressed by the edges of the box plot, is reduced in 'SPM12 + SS' and 'MRTool' compared to the others 


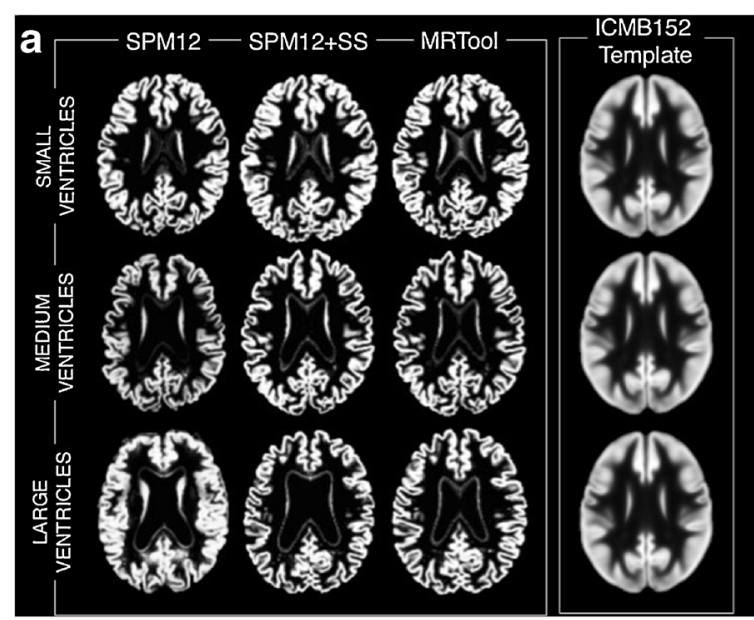

Fig. 6 Tissue segmentation: qualitative analysis. Normalized tissue probability maps (TPMs) of gray matter (a) and white matter (b) of three representative subjects (top row: female 75.4 years old, middle row: female 80.1 years old, bottom row: male 70.4 years old) with increasing

values than 'SPM12 + SS' for MR images characterized by relatively large ventricular size. In this respect, differences between methods were more evident for WM than for GM. However, it is worth noting that our method was not able to adequately register the periventricular WM in the case of ventricles of largest size.

\section{Discussion}

Spatial registration is a fundamental step for both structural and functional investigations allowing the transformation of brains from an individual to a common anatomical space. The process of establishing such a spatial correspondence across subjects becomes particularly difficult in the case of MR images collected in elderly individuals. Our analysis revealed that the unified registration-segmentation approach of SPM12 may not deliver an accurate normalization in the case of subjects with a marked ventricular enlargement. The results suggested that a considerable improvement of the method may be obtained implementing a brain extraction procedure prior the registration/segmentation. However, our proposed solution generally showed superior performance to the standard SPM12 approach, and the improvement in performance was particularly evident in the most difficult conditions for spatial registration, i.e. when brain anatomy was substantially different from the template.

\section{Main Features of our Approach}

The hallmark of 'MRTool' is the use of a registration step specifically aimed at partially correcting gross deviations between the individual MR image and the template, without impacting on local image features. For this step, an

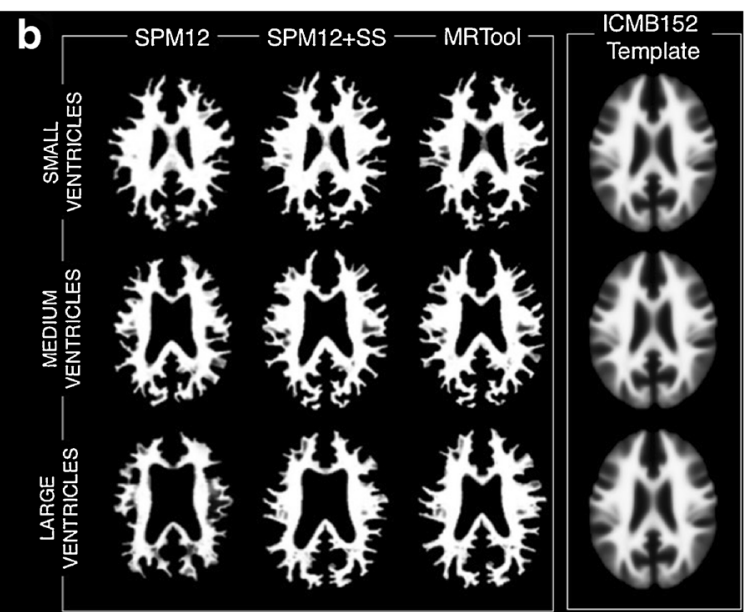

lateral ventricles volume $(20 \mathrm{~mL}, 45.9 \mathrm{~mL}, 81.3 \mathrm{~mL})$ are shown. From left to right: TPMs obtained using the 'SPM12', 'SPM12 + SS', 'MRTool' and the reference TPMs associated with the ICBM152-MNI T1-w template

intensity-based parcellation is used to generate a clustered image that highlights the spatial boundaries between different tissues (Fig. 2). Our intensity-based parcellation approach is substantially different from the approach implemented in SPM12, where the images are segmented into different classes using a mixture model algorithm that identified voxels assigned to a tissue type combined with a priori knowledge (tissue priors) of their spatial distribution. Notably, brain anatomy of elderly individuals often shows a considerable spatial variability (Salonen et al. 1997), especially around the lateral ventricles, and SPM12 tissue probability maps (tissue priors) may not adequately account for such variability. By implementing a coarse registration, defined in the context of the intensity-based parcellation, we tried to overcome such limitation. The use of clustered images in place of the actual MR images, which characterizes the coarse registration step, yields an enhancement in image contrast. Accordingly, the boundaries between GM, WM and CSF are more clearly defined than in actual MR images. This permits a more accurate nonlinear spatial registration (Ashburner and Friston 1999), and specifically leads to the correction of gross differences between the individual and the template MR image.

Once the overall brain morphology has been corrected by using the registration step discussed above, skull-stripping is applied. In the unified registration-segmentation approach of SPM12, skull-stripping can be implicitly attained by a weighting of non-brain tissues (skull, soft tissues and background) with specific tissue probability maps. Instead, 'MRTool' implements an explicit skull-stripping step, for which a brain mask is calculated and applied to the MR image. This choice is based on several studies reporting a clear improvement in the registration process when skull-stripped images are used (Fein et al. 2006; Acosta-Cabronero et al. 2008; Fischmeister et al. 2013). After skull-stripping, the unified 


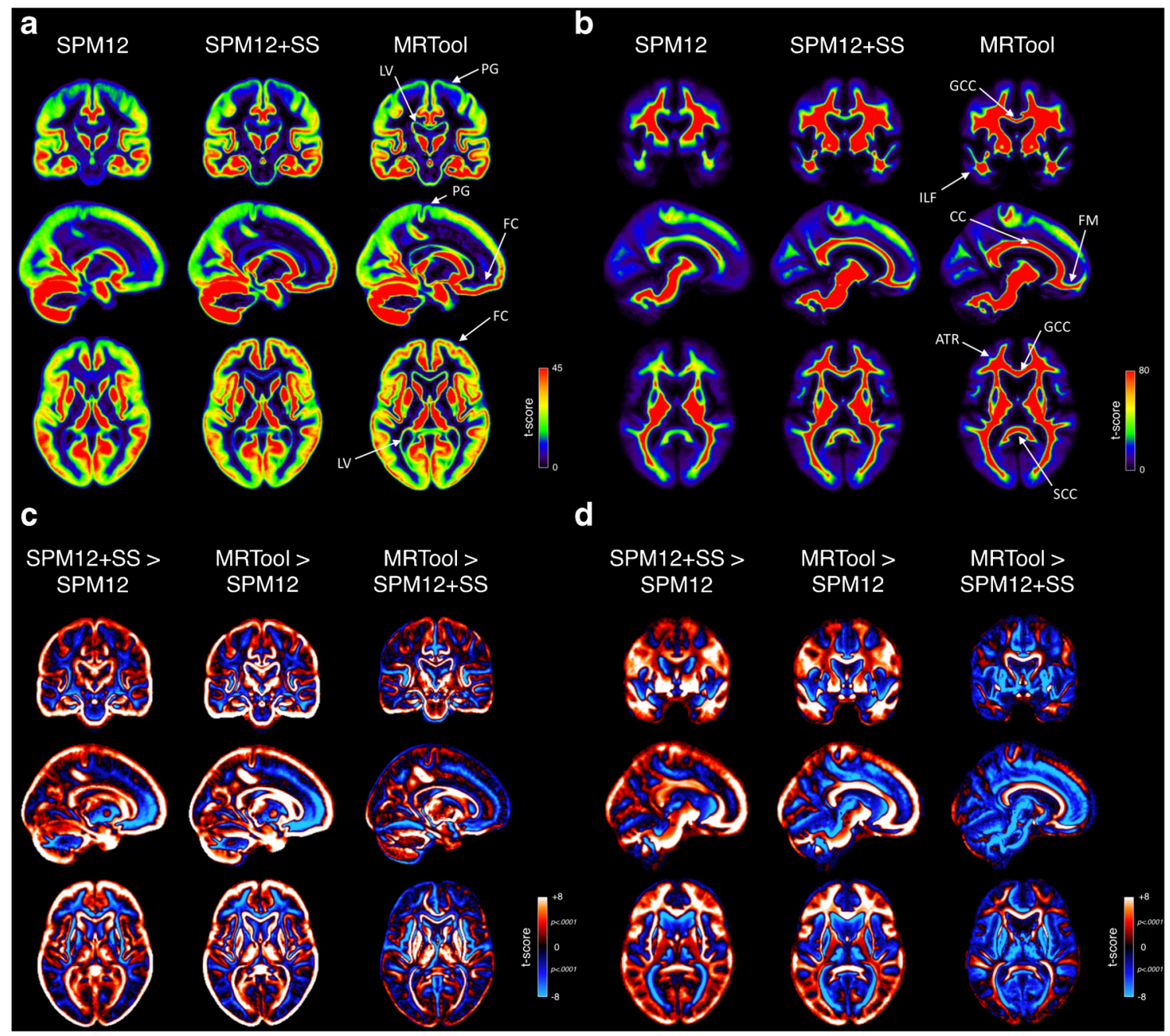

Fig. 7 Within- and between-method analysis of GM and WM probability maps. The within- (a-b) and between-method across-subject analyses (c-d) of GM and WM probability maps were conducted on a voxel-wise basis. The maps show t-values (obtained using nonparametric one-sample and paired t-tests, respectively) over the same cut-planes (coronal, sagittal and axial) for GM and WM, respectively. Higher tscore values obtained from within-method analyses indicate a relatively

registration-segmentation algorithm of SPM12 is run, so that a final normalized image and tissue maps in template space are generated. Not only does this last registration step provide a local fine-tuning in brain anatomy, but also leads to a potential improvement in tissue segmentation. Having a brain closely matching the template priors may ease the tissue classification, which in turn improves the final registration.

\section{Comparison with Other Approaches}

Normalization (Figs. 4 and 5) and segmentation (Fig. 6) quality can be different depending on the image processing steps used. Accordingly, we investigated different setups based on SPM12. This provided a consistent benchmark for the assessment with our proposed improved spatial alignment between subjects. This was the case for the following brain regions: precentral gyrus (PG), lateral ventricles (LV), frontal cortex (FC), genu of corpus callosum (GCC), inferior longitudinal fasciculus (ILF), forceps minor (FM), anterior thalamic radiation (ATR), splenium of corpus callosum (SCC). Uncorrected $\mathrm{p}$ values are reported on the right side of the colorbar

approach. Specifically, we evaluated the registration and segmentation outputs with two different approaches (Fig. 3 ). Not only did this evaluation allow us to identify the most appropriate processing framework to be used in SPM12, but also laid the groundwork for our method.

We observed a clear superiority in the performance of 'SPM12 + SS' with respect to 'SPM12', for both spatial registration and segmentation (Figs. 4 and 6). The use of 'SPM12 + SS' also yield smaller inter-subject variability across images registered to template space (Fig. 7). We argue that this improvement is associated with the removal of nonbrain structures from MR image, which is part of the analysis pipeline of 'SPM12 + SS'. The enhanced performance of the latter as compared to the standard setup corroborates our choice to include a brain masking step in 'MRTool'. 
a

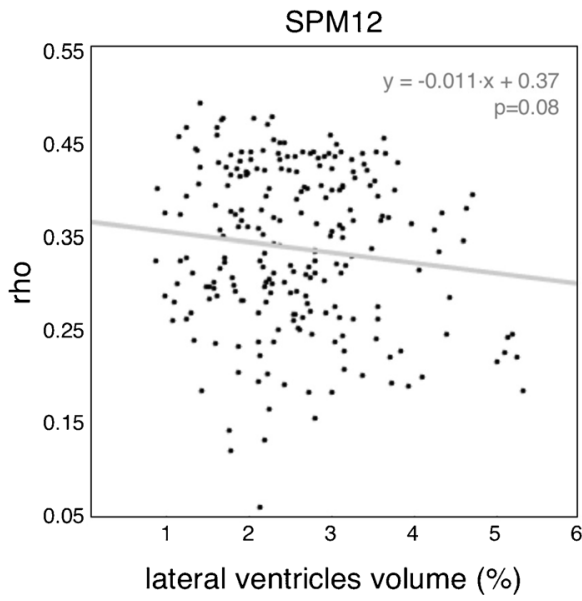

b

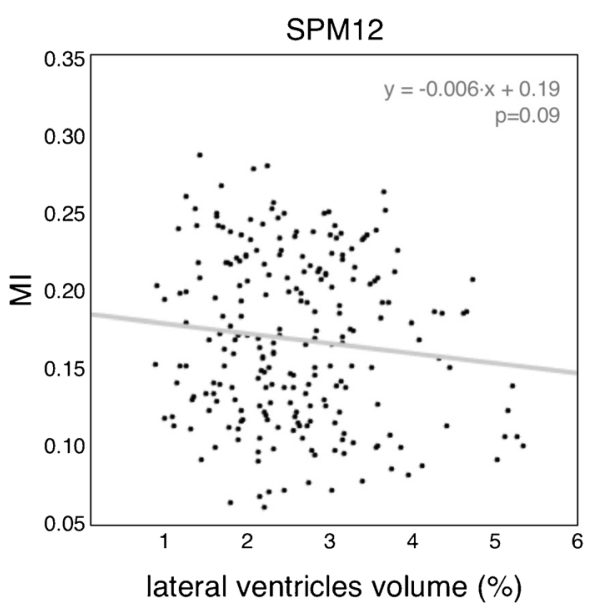

Spatial correlation

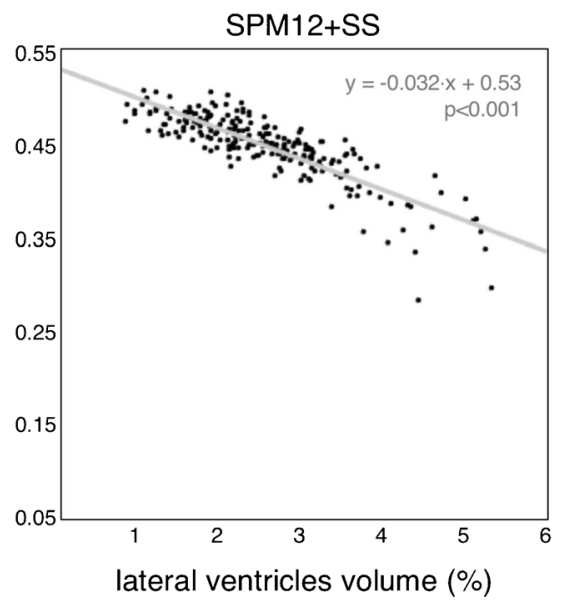

Mutual Information

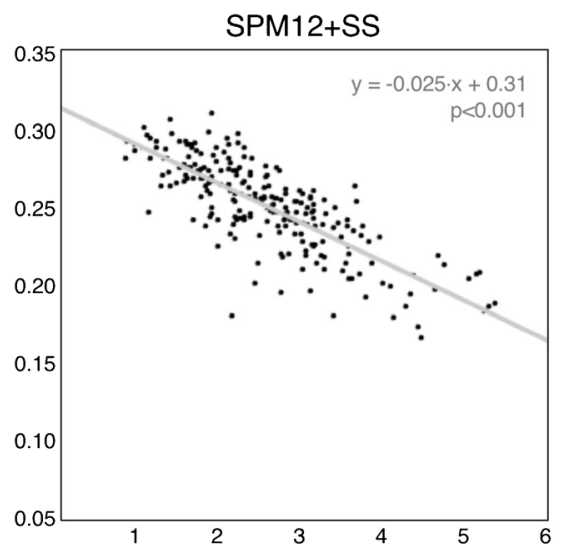

lateral ventricles volume (\%)
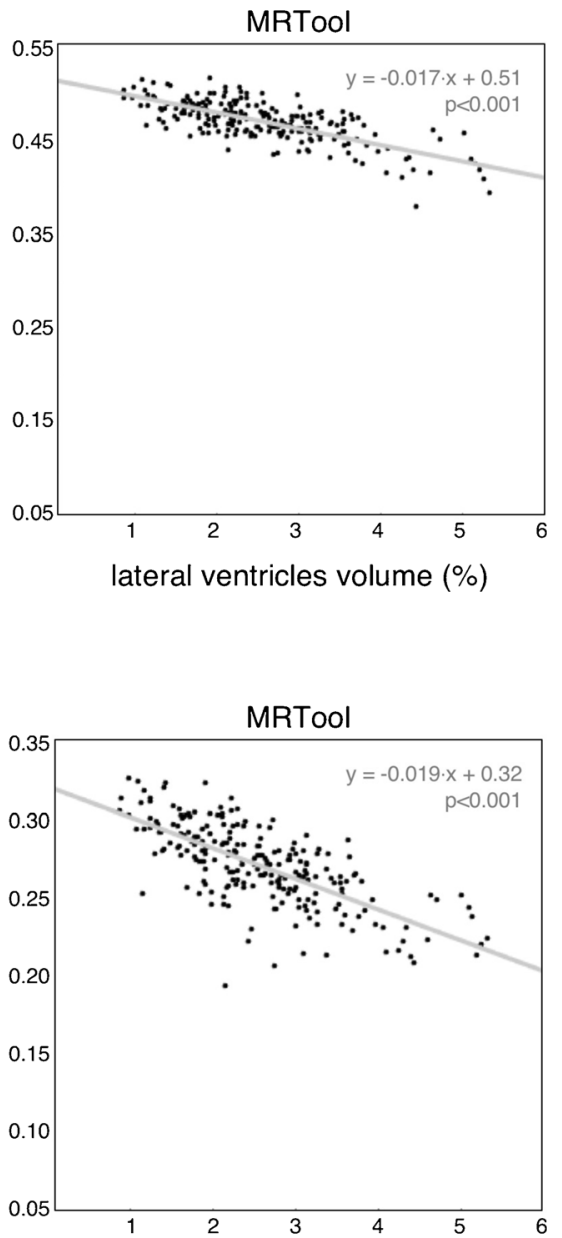

lateral ventricles volume (\%)
Fig. 8 Spatial registration performance with respect to ventricular volume. These scatterplots show the voxel-wise CC (a) and MI (b) between the normalized T1-weighted (T1-w) image and the reference ICBM152-MNI T1-w template for the two different SPM12 setups ('SPM12' and 'SPM12 + SS') and our approach ('MRTool'). The estimated regression line is shown along with the respective equation and $\mathrm{p}$ -

As previously mentioned, we posit that the main improvement of 'MRTool' over the other approaches is likely to be the use of a nonlinear registration step that specifically addresses the problem of large ventricles. Indeed, we observed that the improvement in performance of 'MRTool' compared to the other two setups was superior with MR images collected in individuals with enlarged lateral ventricles (Fig. 8). Likewise, the inter-subject overlap between segmentations (Fig. 9) and the voxel-wise analysis of tissue maps (Fig. 7) provided converging evidence for an increased reproducibility in tissue classification across subjects with marked anatomical variability.

\section{Caveats}

Some limitations in the current study should be considered. First, we evaluated the unified registration-segmentation value. The inter-subject variability, expressed by the dispersion around the line, is reduced in 'SPM12 + SS' and 'MRTool' compared to 'SPM12', regardless of the performance metric used. Although a decline in performance related to the later ventricle size is evident for all methods, 'MRTool' had superior performance than 'SPM12 + SS' and 'SPM12', in particular for brain images with large ventricles

method using default parameters. These settings, although recommended by the authors, may not be optimal for MR images collected in elderly individuals, which are typically characterized by brain atrophy and enlarged ventricles. Nevertheless, there is established consensus that with sufficiently large degrees of freedom, a nonlinear registration can optimally fit any image with a given template. This poses a serious risk of losing anatomical specificity. For this reason, we decided to use default settings for both coarse registration and unified registration-segmentation. Another potential limitation relates to the template used in the study. This template represents middle-aged healthy individuals with normal brain anatomy. It should be noted that we would have most likely obtained better registration performance by using an MR template that is age-matched to the study participants. Nonetheless, we decided to use the standard template 
Fig. 9 Inter-subject segmentation overlap. The performance of the segmentation was assessed comparing tissue probability maps (TPM) across subjects. Specifically, the Dice Similarity index (DSI) was computed on the normalized TPM of gray matter (a) and white matter (b). The probabilities were thresholded at 0.5 to compute the number of misclassifications.

Values range from zero to one, where a higher DSI indicates complete overlap. The computation was iterated for each pair of subjects resulting in a m-by-m matrix with $m$ equals to the total number of subjects (sorted in ascending order with respect to the relative ventricular volume, $\mathrm{SV}$ : small ventricles, LV: large ventricles). The same calculation was then repeated for each method a Gray Matter segmentation: Inter-subject overlap
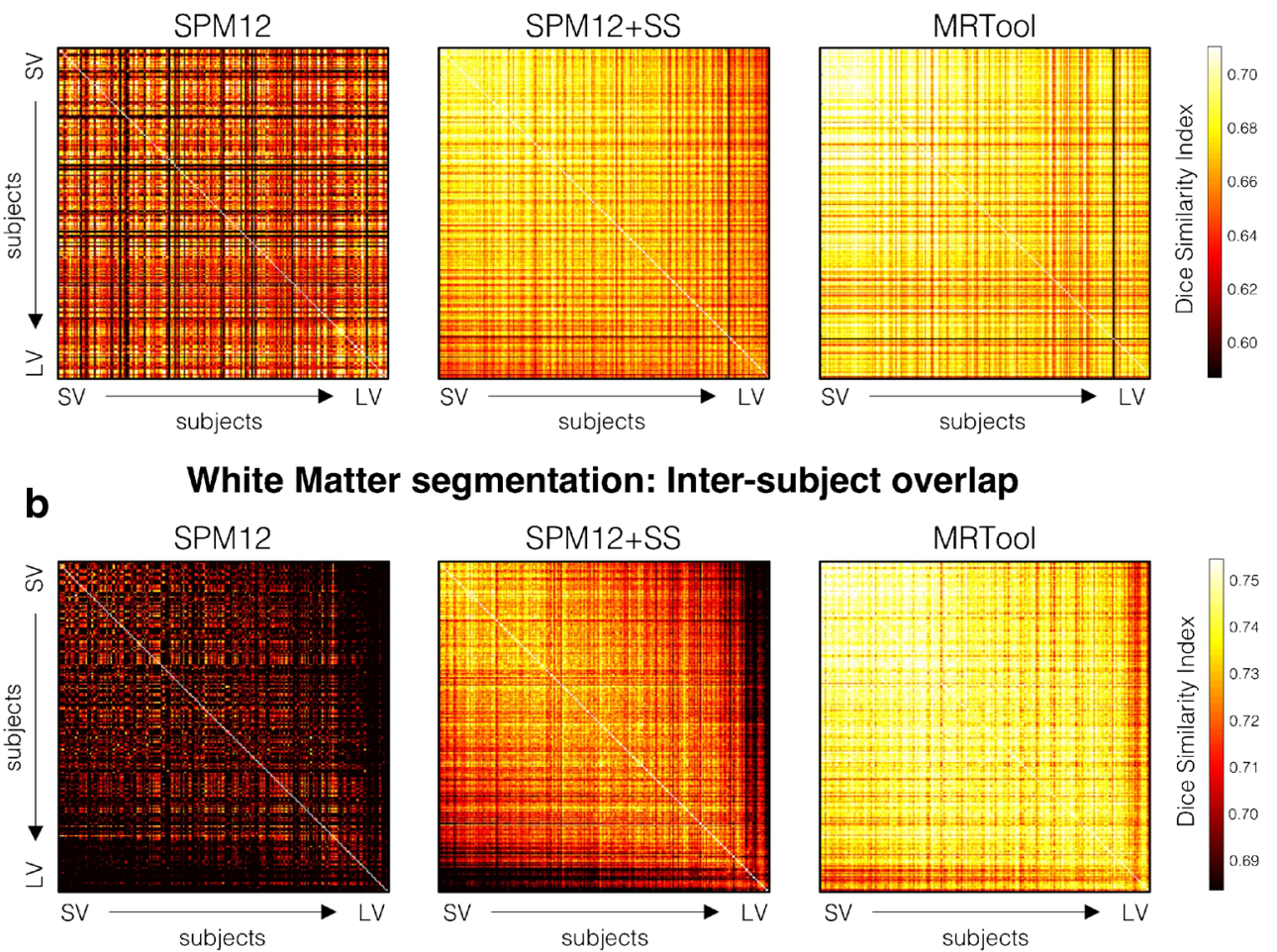

subjects

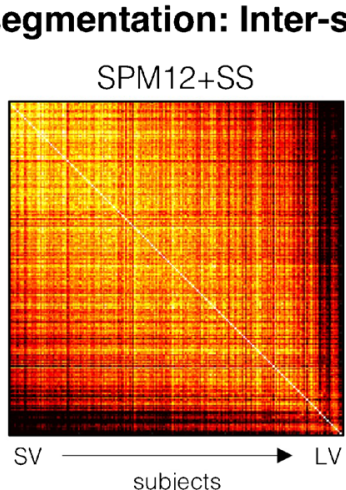

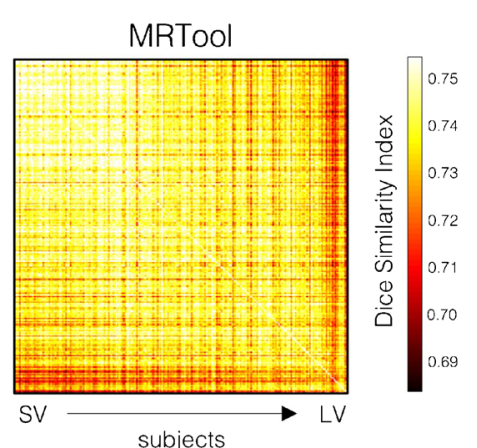

available in SPM12 to facilitate comparisons with other studies. This might have hindered the detection of specific differences between the spatial registration approaches under investigation. Another important limitation relates to the performance measures adopted. Spatial alignment between two images was measured by $\mathrm{CC}$, which can however assess only linear relationships and is sensitive to outliers. To account for this potential problem, we have relied also on the MI measure. Since MI makes no assumptions regarding the nature of the relation between the image intensities, it is considered more robust than CC. Furthermore, although CC and MI measures rely on information directly extracted from the image, there is no consensus on their sensitivity in detecting an accurate spatial registration. This is the reason why we complemented our performance analysis by evaluating the segmentation results in terms of DSI. The evaluation of image alignment on the basis on manually labeled brain regions may be a reliable measure of spatial registration accuracy (Klein et al. 2009). However, this approach could not be adopted in our study, as no manual labelling of brain regions is currently available for MR data collected in older individuals.

\section{Conclusions}

A precise spatial registration is a crucial step to investigate structural changes in normal aging as well as across a wide spectrum of brain pathologies. Our results suggest that

caution should be taken when assessing morphological structure in elderly subjects, particularly in subcortical regions around the ventricles. To address the issue of spatial registration of MR images from older individuals, we proposed an approach that can yield significant improvements as compared to other approaches based on SPM12. The developed tool is freely available and integrated in our software toolbox MRTool (http://www.nitrc.org/projects/ mrtool/). We hope that our work will contribute to a more widespread use of robust and automated approaches for large-scale analyses of structural MR data.

\section{Information Sharing Statement}

All the subjects included in this study were extracted from the publically available Alzheimer's Disease Neuroimaging Initiative (ADNI) dataset (RRID:SCR 003007, http://adni. loni.usc.edu). ADNI is a longitudinal, multicenter study with the goal of developing clinical, imaging, genetic, and biochemical biomarkers for the early detection and tracking of Alzheimer's disease (AD). It comprises subjects with early mild cognitive impairment, with early $A D$, and normal controls. The method developed in this study is freely available and distributed as an independent module in our software toolbox MRTool (RRID:SCR_015956, http://www.nitrc.org/projects/mrtool/). Different setups based on SPM12 (RRID:SCR_007037, http://www.fil.ion. 
ucl.ac.uk/spm/software/spm12/) were used as benchmark for the assessment of our approach. Statistical maps were generated using FSL (RRID:SCR_002823, https://fsl.fmrib. ox.ac.uk/fsl/fslwiki). The manual segmentation of the lateral ventricles on the individual's T1-w image was performed using the open-source software, ITK-SNAP (RRID:SCR 002010, http://www.itksnap.org).

Acknowledgements Data collection and sharing for this project was funded by the Alzheimer's Disease Neuroimaging Initiative (ADNI) (National Institutes of Health Grant U01 AG024904) and DOD ADNI (Department of Defense award number W81XWH-12-2-0012). ADNI is funded by the National Institute on Aging, the National Institute of Biomedical Imaging and Bioengineering, and through generous contributions from the following: AbbVie, Alzheimer's Association; Alzheimer's Drug Discovery Foundation; Araclon Biotech; BioClinica, Inc.; Biogen; Bristol-Myers Squibb Company; CereSpir, Inc.; Cogstate; Eisai Inc.; Elan Pharmaceuticals, Inc.; Eli Lilly and Company; EuroImmun; F. HoffmannLa Roche Ltd. and its affiliated company Genentech, Inc.; Fujirebio; GE Healthcare; IXICO Ltd.; Janssen Alzheimer Immunotherapy Research \& Development, LLC.; Johnson \& Johnson Pharmaceutical Research \& Development LLC.; Lumosity; Lundbeck; Merck \& Co., Inc.; Meso Scale Diagnostics, LLC.; NeuroRx Research; Neurotrack Technologies; Novartis Pharmaceuticals Corporation; Pfizer Inc.; Piramal Imaging; Servier; Takeda Pharmaceutical Company; and Transition Therapeutics. The Canadian Institutes of Health Research is providing funds to support ADNI clinical sites in Canada. Private sector contributions are facilitated by the Foundation for the National Institutes of Health (www.fnih.org). The grantee organization is the Northern California Institute for Research and Education, and the study is coordinated by the Alzheimer's Therapeutic Research Institute at the University of Southern California. ADNI data are disseminated by the Laboratory for Neuro Imaging at the University of Southern California. Author MG received funding from the FWO and European Union's Horizon 2020 research and innovation programme under the Marie Skłodowska-Curie grant agreement No: 665,501.

\section{References}

Acosta-Cabronero, J., Williams, G. B., Pereira, J. M. S., Pengas, G., \& Nestor, P. J. (2008). The impact of skull-stripping and radiofrequency bins correction on grey-matter segmentation for voxelbased morphometry. NeuroImage, 39, 1654-1665.

Allen, J. S., Bruss, J., Mehta, S., Grabowski, T., Brown, C. K., \& Damasio, H. (2008). Effects of spatial transformation on regional brain volume estimates. NeuroImage, 42, 535-547.

Apostolova, L. G., Green, A. E., Babakchanian, S., Hwang, K. S., Chou, Y. Y., Toga, A. W., \& Thompson, P. M. (2012). Hippocampal atrophy and ventricular enlargement in normal aging, mild cognitive impairment (MCI), and Alzheimer disease. Alzheimer Disease and Associated Disorders, 26, 17-27.

Ashburner, J., \& Friston, K. J. (1999). Nonlinear spatial normalization using basis functions. Human Brain Mapping, 7, 254-266.

Ashburner, J., \& Friston, K. J. (2005). Unified segmentation. NeuroImage, 26, 839-851.

Brett, M., Johnsrude, I. S., \& Owen, A. M. (2002). The problem of functional localization in the human brain. Nature Reviews Neuroscience, 3, 243-249.

Collignon, A., Maes, F., Delaere, D., Vandermeulen, D., Suetens, P., \& Marchal, G. (1995). Automated multi-modality image registration based on information theory. Information Processing in Medical Imaging, 3, 263-274.
Colloby, S. J., Firbank, M. J., Vasudev, A., Parry, S. W., Thomas, A. J., \& O'brien, J. T. (2011). Cortical thickness and VBM-DARTEL in latelife depression. Journal of Affective Disorders, 133, 158-164.

Crivello, F., Schormann, T., Tzourio-Mazoyer, N., Roland, P. E., Zilles, K., \& Mazoyer, B. M. (2002). Comparison of spatial normalization procedures and their impact on functional maps. Human Brain Mapping, 16, 228-250.

Dade, L. A., Gao, F. Q., Kovacevic, N., Roy, P., Rockel, C., O'toole, C. M., Lobaugh, N. J., Feinstein, A., Levine, B., \& Black, S. E. (2004). Semiautomatic brain region extraction: A method of parcellating brain regions from structural magnetic resonance images. NeuroImage, 22, 1492-1502.

Dewey, J., Hana, G., Russell, T., Price, J., Mccaffrey, D., Harezlak, J., Sem, E., Anyanwu, J. C., Guttmann, C. R., Navia, B., Cohen, R., Tate, D. F., \& Consortium, H. N. (2010). Reliability and validity of MRI-based automated volumetry software relative to auto-assisted manual measurement of subcortical structures in HIV-infected patients from a multisite study. NeuroImage, 51, 1334-1344.

Dong, L., \& Boyer, A. L. (1995). An image correlation procedure for digitally reconstructed radiographs and electronic portal images. International Journal of Radiation Oncology Biology Physics, 33, 1053-1060.

Eloyan, A., Shou, H. C., Shinohara, R. T., Sweeney, E. M., Nebel, M. B., Cuzzocreo, J. L., Calabresi, P. A., Reich, D. S., Lindquist, M. A., \& Crainiceanu, C. M. (2014). Health effects of lesion localization in multiple sclerosis: Spatial registration and confounding adjustment. PLoS One, 9, e107263.

Fein, G., Landman, B., Tran, H., Barakos, J., Moon, K., Di Sclafani, V., \& Shumway, R. (2006). Statistical parametric mapping of brain morphology: Sensitivity is dramatically increased by using brainextracted images as inputs. NeuroImage, 30, 1187-1195.

Fischl, B., Salat, D. H., Busa, E., Albert, M., Dieterich, M., Haselgrove, C., Van Der Kouwe, A., Killiany, R., Kennedy, D., Klaveness, S., Montillo, A., Makris, N., Rosen, B., \& Dale, A. M. (2002). Whole brain segmentation: Automated labeling of neuroanatomical structures in the human brain. Neuron, 33, 341-355.

Fischmeister, F. P. S., Hollinger, I., Klinger, N., Geissler, A., Wurnig, M. C., Matt, E., Rath, J., Robinson, S. D., Trattnig, S., \& Beisteiner, R. (2013). The benefits of skull stripping in the normalization of clinical fMRI data. Neuroimage Clinical, 3, 369-380.

Ganzetti, M., Wenderoth, N., \& Mantini, D. (2015). Quantitative evaluation of intensity inhomogeneity correction methods for structural MR brain images. Neuroinformatics, 14, 5-21.

Ganzetti, M., Wenderoth, N., \& Mantini, D. (2016). Intensity inhomogeneity correction of structural MR images: A data-driven approach to define input algorithm parameters. Frontiers in Neuroinformatics, 10, 10.

Han, Z., Thornton-Wells, T. A., Dykens, E. M., Gore, J. C., \& Dawant, B. M. (2012). Effect of nonrigid registration algorithms on deformation-based morphometry: A comparative study with control and Williams syndrome subjects. Magnetic Resonance Imaging, 30, 774-788.

Hoeksma, M. R., Kenemans, J. L., Kemner, C., \& Van Engeland, H. (2005). Variability in spatial normalization of pediatric and adult brain images. Clinical Neurophysiology, 116, 1188-1194.

Izquierdo-Garcia, D., Hansen, A. E., Forster, S., Benoit, D., Schachoff, S., Furst, S., Chen, K. T., Chonde, D. B., \& Catana, C. (2014). An SPM8based approach for attenuation correction combining segmentation and nonrigid template formation: Application to simultaneous PET/MR brain imaging. Journal of Nuclear Medicine, 55, 1825-1830.

Kennedy, K. M., Erickson, K. I., Rodrigue, K. M., Voss, M. W., Colcombe, S. J., Kramer, A. F., Acker, J. D., \& Raz, N. (2009). Age-related differences in regional brain volumes: A comparison of optimized voxel-based morphometry to manual volumetry. Neurobiology of Aging, 30, 1657-1676.

Klein, A., Andersson, J., Ardekani, B. A., Ashburner, J., Avants, B., Chiang, M. C., Christensen, G. E., Collins, D. L., Gee, J., Hellier, P., Song, J. H., Jenkinson, M., Lepage, C., Rueckert, D., Thompson, P., Vercauteren, T., Woods, R. P., Mann, J. J., \& Parsey, R. V. (2009). 
Evaluation of 14 nonlinear deformation algorithms applied to human brain MRI registration. NeuroImage, 46, 786-802.

Kleinbaum, D. G., Kupper, L. L., Nizam, A., \& Muller, K. E. (2007). Applied regression analysis and other multivariable methods. Belmont: Duxbury Press.

Lemieux, L., Jagoe, R., Fish, D. R., Kitchen, N. D., \& Thomas, D. G. T. (1994). A patient-to-computed-tomography image registration method based on digitally reconstructed radiographs. Medical Physics, 21, 1749-1760.

Maes, F., Collignon, A., Vandermeulen, D., Marchal, G., \& Suetens, P. (1997). Multimodality image registration by maximization of mutual information. IEEE Transactions on Medical Imaging, 16, 187-198.

Martino, M. E., De Villoria, J. G., Lacalle-Aurioles, M., Olazaran, J., Cruz, I., Navarro, E., Garcia-Vazquez, V., Carreras, J. L., \& Desco, M. (2013). Comparison of different methods of spatial normalization of FDG-PET brain images in the voxel-wise analysis of $\mathrm{MCI}$ patients and controls. Annals of Nuclear Medicine, 27, 600-609.

Mcdonald, C. R., Mcevoy, L. K., Gharapetian, L., Fennema-Notestine, C., Hagler, D. J., Holland, D., Koyama, A., Brewer, J. B., Dale, A. M., \& Initi, A. S. D. N. (2009). Regional rates of neocortical atrophy from normal aging to early Alzheimer disease. Neurology, 73, 457-465.

Pallant, J. (2013). SPSS survival manual: A step by step guide to data analysis using IBM Spss. Allen \& Unwin.

Peelle, J. E., Cusack, R., \& Henson, R. N. A. (2012). Adjusting for global effects in voxel-based morphometry: Gray matter decline in normal aging. NeuroImage, 60, 1503-1516.

Petersen, R. C., Aisen, P. S., Beckett, L. A., Donohue, M. C., Gamst, A. C., Harvey, D. J., Jack Jr., C. R., Jagust, W. J., Shaw, L. M., Toga, A. W., Trojanowski, J. Q., \& Weiner, M. W. (2010). Alzheimer's disease neuroimaging initiative (ADNI): Clinical characterization. Neurology, 74, 201-209.

Pohl, K. M., Fisher, J., Levitt, J. J., Shenton, M. E., Kikinis, R., Grimson, W. E. L., \& Wells, W. M. (2005). A unifying approach to registration, segmentation, and intensity correction. Medical Image Computing and Computer Assisted Intervention, 8(Pt 1), 310-318.
Pu, F., Xu, L. Q., Li, D. Y., Fan, Y. B., Niu, H. J., \& Li, S. Y. (2013). Comparison of two nonlinear registration techniques to investigate brain atrophy patterns in normal aging. Journal of Neuroradiology, 40, 326-334.

Salonen, O., Autti, T., Raininko, R., Ylikoski, A., \& Erkinjuntti, T. (1997). MRI of the brain in neurologically healthy middle-aged and elderly individuals. Neuroradiology, 39, 537-545.

Shattuck, D. W., Sandor-Leahy, S. R., Schaper, K. A., Rottenberg, D. A., \& Leahy, R. M. (2001). Magnetic resonance image tissue classification using a partial volume model. NeuroImage, 13, 856-876.

Van Leemput, K., Maes, F., Vandermeulen, D., \& Suetens, P. (1999a). Automated model-based bias field correction of MR images of the brain. IEEE Transactions on Medical Imaging, 18, 885-896.

Van Leemput, K., Maes, F., Vandermeulen, D., \& Suetens, P. (1999b). Automated model-based tissue classification of MR images of the brain. IEEE Transactions on Medical Imaging, 18, 897-908.

Weiner, M. W., Veitch, D. P., Aisen, P. S., Beckett, L. A., Cairns, N. J., Green, R. C., Harvey, D., Jack, C. R., Jagust, W., Liu, E., Morris, J. C., Petersen, R. C., Saykin, A. J., Schmidt, M. E., Shaw, L., Siuciak, J. A., Soares, H., Toga, A. W., Trojanowski, J. Q., \& Alzheimer's Disease Neuroimaging, I. (2012). The Alzheimer's disease neuroimaging initiative: A review of papers published since its inception. Alzheimers Dement, 8, S1-68.

Wells 3rd, W. M., Viola, P., Atsumi, H., Nakajima, S., \& Kikinis, R. (1996). Multi-modal volume registration by maximization of mutual information. Medical Image Analysis, 1, 35-51.

Whitwell, J. L., Shiung, M. M., Przybelski, S. A., Weigand, S. D., Knopman, D. S., Boeve, B. F., Petersen, R. C., \& Jack, C. R. (2008). MRI patterns of atrophy associated with progression to $\mathrm{AD}$ in amnestic mild cognitive impairment. Neurology, 70, 512-520.

Wilke, M., Schmithorst, V. J., \& Holland, S. K. (2002). Assessment of spatial normalization of whole-brain magnetic resonance images in children. Human Brain Mapping, 17, 48-60. 